Original Research

\title{
Prevalence of Radiographic Signs of Osteoarthritis in Lusitano Purebred Horses
}

\author{
Sofia Ramos ${ }^{\mathrm{a}, ~}{ }^{*}$, António Pinto a , Madalena Cardoso a, Nuno Alexandre a \\ Elisa Bettencourt ${ }^{\mathrm{a}}$, Susana Monteiro ${ }^{\mathrm{a}}$, Luis Telo Gama ${ }^{\mathrm{b}}$ \\ a Veterinary Medicine Department, MED - Mediterranean Institute for Agriculture, Environment and Development, School of Science and Technology, \\ University of Évora, Évora, Portugal \\ ${ }^{\mathrm{b}}$ Veterinary Medicine Faculty, CIISA - Center for Interdisciplinary Research in Animal Health, University of Lisbon, Avenida da Universidade Técnica, Lisboa, \\ Portugal
}

\section{A R T I C L E I N F O}

\section{Article history:}

Received 27 April 2020

Received in revised form

15 July 2020

Accepted 16 July 2020

Available online 23 July 2020

\section{Keywords:}

Horse

Radiograph

Osteoarthritis

Prevalence

Correlation

Joint

\begin{abstract}
A B S T R A C T
Radiographic prevalence and correlation of radiographic findings has not been performed in Lusitano Purebred horses. The aim of this study was to (1) evaluate the prevalence of primary osteoarthritis radiographic findings in Lusitano Purebred horses; (2) to assess correlations between radiographic findings in different joints of the same limb and different limbs; and (3) elucidate the effect of age in the radiographic findings. A radiographic protocol of the stifle, tarsi, fetlocks and distal limbs was done in 98 Lusitanos and the classification of the radiographs was performed using a 0-4 scale developed and applied blindly by three veterinarians. The distal interphalangeal, proximal interphalangeal, metacarpophalangeal, metatarsophalangeal, tarsometatarsal, distal intertarsal, proximal intertarsal/, tibiotarsal and femorotibial-patellar joints were evaluated. Most joints presented no abnormal findings or minor abnormal radiographic findings ( $82.86 \%$ grade $\leq 1)$. The most affected joint was tarsometatarsal and more severe lesions were found in tarsometatarsal and distal intertarsal. Femorotibial-patellar radiographic changes were rare (2.13\%). A strong/moderate correlation was found between contralateral joints with exception hindlimb fetlocks. A moderate correlation was found between fore and hindlimbs for distal limb joints. When analyzing ipsilateral as well as diagonal distal limbs, a strong/ moderate correlation was also found. The total score progressed in 0.2 score points per each year of age, revealing that age can be a statistically significant predictor for radiographic changes. Overall, Lusitano horses presented a low prevalence of severe radiographic sings of primary osteoarthritis. Findings in contralateral joints tend to be correlated.
\end{abstract}

() 2020 Elsevier Inc. All rights reserved.

\section{Introduction}

Osteoarthritis $(\mathrm{OA})$ is one of the most commonly referred causes of lameness in horses as a result of pain and/or mechanical restrictions [1]. Predisposing factors for primary OA could be related

Author's contribution: S. R. collected data, analyzed and interpreted data, and drafted the article. A. P. collected data, analyzed and interpreted data, and critically reviewed the article. M. C. collected data and interpreted and critically reviewed the article. N. A. designed data collection instruments and critically reviewed the article. E. B. designed the study and data collection instruments, interpreted data and drafted, and critically reviewed the article. S. M. collected data, analyzed data, and critically reviewed the article. L. G. designed the study, analyzed data and performed the statistical analyses, and critically reviewed the article.All authors approved the final version.

Owner Informed Consent: This study was performed with client consent, and the client confidentiality was assured. The figures used in this manuscript cannot be with age or joint overloading as well as conformation, improper shoeing, prolonged joint immobilization, training frequency/intensity, or work surface [2].

The radiographic exam enables the veterinary clinician to detect abnormalities prone to compromise the soundness of the horse [3]

identified specifically.

Animal welfare/Ethical statement: Research ethics committee oversight was not required for this analysis of clinical data. Owners gave consent for the inclusion of their animals' information in the study.

Conflict of interest statement: There are no conflicts of interest (financial, personal, or other relationships with people/organizations) that could inappropriately have influenced our work.

* Corresponding author at: Sofia Ramos, Department of Companion Animals and Equids, University of Liège, Liège, Belgium.

E-mail address: ascramos@uliege.be (S. Ramos). 
and even its future athletic performance [4-6]. However, radiographs may not be able to detect early stages of $\mathrm{OA}$, and a poor correlation between radiographic findings and clinical signs of this disease has been reported by several authors (especially when only low-grade radiographic changes are found) $[1,4,7]$. Despite this fact, the radiographic diagnostic method remains widely available and used and therefore, studies about radiographic changes are still required in many disciplines and breeds.

The Lusitano horse is an extremely versatile sport horse and is the most important native equine breed in Portugal. Radiographic exams are performed routinely by clinicians, however, there is a lack of studies concerning the overall prevalence of OA in Lusitano horses. Further investigation is imperative to ensure a rational use of Lusitano sport horses, to improve Lusitano Purebred horses' potential through selection and breeding programs. Moreover, the objective evaluation of radiographic changes using a scoring system developed from other studies [8,9] is crucial to perform comparative studies.

The purpose of this study is to: 1) evaluate the prevalence of primary OA radiographic findings in Lusitano Purebred horses, 2) assess correlations between radiographic findings in different joints of the same limb and different limbs of the same horse, and 3) elucidate the effect of age in radiographic findings.

\section{Material and Methods}

\subsection{Examined Horse Population, Age, and Disciplines}

All horses included in this study were Lusitano Purebred ( $\mathrm{n}=$ 98), from the mid-south regions of Portugal presented for a radiographic exam between July 2013 and September 2018.

The horses underwent a radiographic exam including the four distal limbs and both tarsal regions. Forty-seven of the 98 horses examined (47.9\%), underwent a radiographic exam that also included both stifle regions.

The radiographic exams were requested by owners regardless if the horse was lame at the time of the evaluation, mainly for possible future sale. A systematic orthopedic exam with assessment of pain location was not performed, as this was not required by the owners.

Of the 98 horses assessed, 83 were stallions (84.7\%), 14 were females (14.3\%), and one was gelding (1\%). The age ranged between 3 and 25 year old, and the mean age was $7.45 \pm 5.64$ years, with the majority of the population $71.43 \%$ (70/98) under 6 year old. Of the total number of 98 horses, at the time of the exam 59.2\% (58/98) were still being broken with the intention of being used for dressage, $17.35 \%$ (17/98) performed dressage, $8.16 \%$ (8/98) were breeding mares, and $15.8 \%$ (15/98) were stallions for reproductive and/or leisure use.

\subsection{Radiographic Examination}

All radiographic examinations were performed using a standard procedure. All horses were sedated before the radiographic examination using detomidine $(0.01 \mathrm{mg} / \mathrm{kg}$ IV) combined with butorphanol (0.02 mg/kg IV).

The joints evaluated were as follows: distal interphalangeal (DIP), proximal interphalangeal (PIP), metacarpophalangeal (MCP), metatarsophalangeal (MTP), tarsometatarsal (TMT), distal intertarsal (DIT), proximal intertarsal (PIT), tibiotarsal (TT) and femorotibial-patellar (FTP) joints. For the analyses of correlations, the joints were assessed individually and grouped in two categories: one group named "distal limb" that comprised the DIP, PIP, and MCP/MTP joints and a "tarsi" group that comprised the PIT/TT, DIT, and TMT joints, and the sum of scores assigned to these joints was used in the analyses. Two radiographic projections were performed for evaluation of DIP and PIP (lateromedial (LM) and dorsopalmar/plantar (DP) on a block), two radiographic projections for the fetlock joints (LM and dorsal $10^{\circ}$ proximal-palmarodistal oblique view (D10 $\mathrm{Pr}-\mathrm{PaDiO})$, four radiographic projections for evaluation of tarsal joints (LM, DP, oblique $45^{\circ}$-plantaromedial lateral dorsal (DL45-PMO), and oblique medial $45^{\circ}$-plantarolateral dorsal (DM45-PLO)), and one caudal $60^{\circ}$ lateral-craniomedial oblique view for the stifle joints. Images acquired before 2017 were obtained with a Fujifilm FCR XG-1 indirect computed radiographic system; radiographs acquired since 2017 were taken using a direct radiographic system Orange $9020 \mathrm{HF}$ (EcoRay ) and wireless flat panel $1500 \mathrm{cw}$ (CareRay). The choice of the number of radiographic views and the radiographic projections used was dictated by need to work with young horses difficult to manipulate (still being broken), personnel available during the examinations, and the goal of reducing the irradiation risks for the staff.

\subsection{Radiographic Interpretation}

The severity of OA findings was classified for each joint using a system adapted from the studies by Grauw et al. 2006 [8] and Verwilghen et al. 2009 [9], and the radiographic scores were noted on a sheet. The scoring system is outlined in Table 1, where five severity scores (0-4) were used, such that higher scores indicated greater severity of radiographic changes (Figs. 1-4).

Scoring was performed by three clinicians independently, in a blind manner with regard to each other's findings. The scores attributed to each joint by the various judges were averaged for further analyses, and the mean score was converted to a classification grade, obtained as: grade $0=$ score $<0.50$; grade $1=0.50 \leq$ score $<1.50$; grade $2=1.50 \leq$ score $<2.50$; grade $3=2.50 \leq$ score $<$ 3.50 ; and grade $4 \geq 3.50$ score. Although osteochondrosis dissecans or bone cysts as well as fractures may lead to OA [10,11], and their presence was noted, but they were not taken in consideration for this classification as the main objective of the study was to identify the presence of primary OA. Horses that presented clinical signs or history of septic arthritis were excluded, as these would lead to a deterioration in the radiographic score [12] which would bias the results.

A total score per horse was obtained as the sum of the mean scores for each individual joint (scale 0-72).

\subsection{Statistical Analysis}

Statistical analyses were performed using Statistical Analysis Software (SAS), version 9.4. Frequency tables were obtained to assess the prevalence of radiographic changes by joint, by using the grade attributed to each joint.

The mean scores attributed to the various joints and the total score were assessed by analysis of regression, with a linear model which included the effect of age as continuous variable. The correlations between the score of radiographic changes in different pairs of joints, and the correlation of OA scores for different joints for each limb, were assessed through the Pearson correlation coefficient between the mean scores attributed to the different joints. The correlation coefficient ( $r$ ) was considered weak for values between $0.20<r<0.39$, moderate for $0.40<r<0.59$, strong for $0.60<$ $r<0.79$, and very strong when $r \geq 0.80$.

\section{Results}

\subsection{Prevalence of Radiographic Changes by Joint}

The prevalence of radiographic changes by joint is summarized in Table 2. The majority of the joints evaluated presented no 
Table 1

Classification of radiographic findings with their corresponding attributed severity score.

\begin{tabular}{|c|c|c|}
\hline Region & Radiographic findings & Score \\
\hline \multirow[t]{15}{*}{ Distal interphalangeal (DIP) joint } & $\begin{array}{l}\text { Rounded joint margin of distal phalanx } \\
\text { No subchondral bone sclerosis }\end{array}$ & 0 \\
\hline & Sharp or pointed or double point shape of the extensor process & 1 \\
\hline & Minimal localized subchondal bone sclerosis & \\
\hline & Mild osteophyte(s) or entheseophytes formation on or, remodeling or lucency of the extensor process & 2 \\
\hline & Mild osteophyte(s) or entheseophytes formation on the joint margins & \\
\hline & $\begin{array}{l}\text { Mild remodeling or osteophyte formation on the dorsal cortex of the diaphyseal region of the middle phalanx within the } \\
\text { distal interphalangeal joint capsule }\end{array}$ & \\
\hline & Mild localized subchondral bone sclerosis & \\
\hline & Moderate osteophyte(s) or entheseophytes formation, remodeling or lucency of the extensor process & 3 \\
\hline & Moderate osteophyte(s) or entheseophytes formation on the joint margins & \\
\hline & $\begin{array}{l}\text { Moderate remodeling on the dorsal cortex of the diaphyseal region of the middle phalanx within the distal interphalangeal } \\
\text { joint capsule }\end{array}$ & \\
\hline & Moderate localized subchondral bone sclerosis and/or joint space narrowing & \\
\hline & Severe osteophyte(s) or entheseophytes formation, remodeling or lucency of the extensor process & 4 \\
\hline & $\begin{array}{l}\text { Severe remodeling on the dorsal cortex of the diaphyseal region of the middle phalanx within the distal interphalangeal } \\
\text { joint capsule }\end{array}$ & \\
\hline & Severe osteophyte(s) or entheseophyte(s) on the joint margins & \\
\hline & Severe subchondral bone sclerosis and/or joint space narrowing & \\
\hline \multirow[t]{12}{*}{ Proximal interphalangeal (PIP) joint } & Rounded joint margin of middle phalanx & 0 \\
\hline & No subchondral bone sclerosis & \\
\hline & Sharp or pointed on the dorsoproximal aspect of the middle phalanx or joint margins & 1 \\
\hline & Minimal localized subchondral bone sclerosis & \\
\hline & $\begin{array}{l}\text { Mild periarticular osteophyte(s) or entheseophyte(s) formation or lucency of the dorsoproximal aspect of the middle } \\
\text { phalanx or joint margins }\end{array}$ & 2 \\
\hline & Mild remodeling on the dorsoproximal aspect of the middle phalanx & \\
\hline & Mild localized subchondral bone sclerosis & \\
\hline & $\begin{array}{l}\text { Moderate osteophyte(s) or entheseophyte(s), remodeling or lucency of the dorsoproximal aspect of the middle phalanx or } \\
\text { joint margins }\end{array}$ & 3 \\
\hline & Moderate bone sclerosis of the proximal end of the middle phalanx and/or joint space narrowing & \\
\hline & Severe osteophyte(s) or entheseophyte(s), remodeling or lucency on the dorsoproximal aspect of the middle phalanx & 4 \\
\hline & Severe osteophyte(s) or entheseophyte(s) formation on the joint margins & \\
\hline & Severe subchondral bone sclerosis and/or joint space narrowing & \\
\hline \multirow{7}{*}{$\begin{array}{l}\text { Metacarpophalangeal (MCP) } \\
\text { Metatarsophalangeal } \\
\text { (MTP) joints }\end{array}$} & Rounded joint margins of proximal phalanx & 0 \\
\hline & No subchondral bone sclerosis & \\
\hline & Sharp or pointed joint margins of proximal phalanx or minimal localized subchondral bone sclerosis & 1 \\
\hline & $\begin{array}{l}\text { Mild osteophyte(s) or entheseophyte(s) on joint margins or on the dorso-proximal border of the proximal phalanx, } \\
\text { remodeling or mild localized subchondral bone sclerosis }\end{array}$ & 2 \\
\hline & $\begin{array}{l}\text { Moderate ostheophyte(s) or entheseophyte on joint margins or on the dorso-proximal border of the proximal phalanx or } \\
\text { moderate localized subchondral bone sclerosis and/or joint space narrowing }\end{array}$ & 3 \\
\hline & $\begin{array}{l}\text { Severe osteophyte(s) or entheseophyte(s) formation, severe subchondral bone sclerosis and/or evidence of joint space } \\
\text { narrowing }\end{array}$ & 4 \\
\hline & Severe osteophyte(s) or entheseophyte(s) on joint margins & \\
\hline \multirow[t]{7}{*}{ Tarsometatarsal (TMT) joint } & Rounded joint margin & 0 \\
\hline & No subchondral bone sclerosis & \\
\hline & $\begin{array}{l}\text { Sharp or pointed joint margin on the dorsoproximal aspect of the third metatarsal bone or dorsodistal third tarsal bone with } \\
\text { no subchondral bone sclerosis or joint space narrowing }\end{array}$ & 1 \\
\hline & Prominent III tarsal bone or central tarsal bone & \\
\hline & Mild osteophyte(s) or entheseophyte(s) formation on joint margins and/or mild localized subchondral bone sclerosis & 2 \\
\hline & $\begin{array}{l}\text { Moderate osteophyte(s) or entheseophyte(s) formation on the dorsoproximal aspect of the third metatarsal bone or } \\
\text { dorsodistal aspect of the third tarsal bone or joint margins and/or moderate localized subchondral bone sclerosis and/or joint } \\
\text { space narrowing }\end{array}$ & 3 \\
\hline & $\begin{array}{l}\text { Severe osteophyte(s) or entheseophyte(s) formation on the dorsoproximal aspect of the third metatarsal bone or dorsodistal } \\
\text { third tarsal bone or joint margins with or without lucent areas in the subchondral bone, severe subchondral bone sclerosis, } \\
\text { and/or evidence of joint space narrowing }\end{array}$ & 4 \\
\hline \multirow[t]{8}{*}{ Distal intertarsal (DIT) joint } & Rounded joint margin & 0 \\
\hline & No subchondral bone sclerosis & \\
\hline & Pointed joint margins and/or minimal localized subchondral bone sclerosis & 1 \\
\hline & Mild periarticular osteophyte(s) or entheseophyte(s) formation on the central tarsal bone or joint margins & 2 \\
\hline & $\begin{array}{l}\text { Moderate osteophyte(s) or entheseophyte(s) and/or dorsal remodeling with mild dorsal collapse and/or moderate localized } \\
\text { subchondral bone sclerosis and/or joint space narrowing }\end{array}$ & 3 \\
\hline & Moderate osteophyte(s) or entheseophyte(s) formation on the joint margins & \\
\hline & $\begin{array}{l}\text { Severe osteophyte(s) or entheseophyte, subchondral bone lysis or lucent areas on the distal dorsal aspect of the central tarsal } \\
\text { bone and/or the proximodorsal aspect of the third tarsal bone and/or evidence of joint narrowing }\end{array}$ & 4 \\
\hline & Severe osteophyte(s) or entheseophyte(s) formation on joint margins & \\
\hline \multirow{6}{*}{$\begin{array}{l}\text { Proximal intertarsal Tibiotarsal } \\
\text { (PIT/TT) joint }\end{array}$} & Rounded joint margin & 0 \\
\hline & No subchondral bone sclerosis & \\
\hline & $\begin{array}{l}\text { Pointed joint margin on the dorsoproximal aspect of the central tarsal bone and/or minimal subchondral bone sclerosis or } \\
\text { trochlear idention }\end{array}$ & 1 \\
\hline & Mild periarticular osteophyte(s) on the dorsal aspect of the central tarsal bone or talus with distally directed bony exostosis & 2 \\
\hline & Moderate osteophyte(s) and/or dorsal remodeling of the central tarsal bone with mild dorsal collapse and thining & 3 \\
\hline & Subchondral bone sclerosis, local lucency in the PIT or talocalcaneal joint & 4 \\
\hline
\end{tabular}


Table 1 (continued)

\begin{tabular}{|c|c|c|}
\hline Region & Radiographic findings & Score \\
\hline \multirow[t]{6}{*}{ Femorotibialpatellar (FTP) joint } & Rounded joint margin & 0 \\
\hline & No subchondral bone sclerosis & \\
\hline & Small depression in the middle third of the groove of the trochlea or mild flattening of the trochlear ridge & 1 \\
\hline & $\begin{array}{l}\text { Mild osteophyte or entheseophyte(s) or remodeling present in the patella/irregularity with remodeling of the trochlear } \\
\text { ridge }\end{array}$ & 2 \\
\hline & $\begin{array}{l}\text { Moderate osteophyte or entheseophyte(s) formation on the tibia or caudal aspect of the femur and/or pointy spikes on the } \\
\text { patellar aspect }\end{array}$ & 3 \\
\hline & $\begin{array}{l}\text { Severe osteophyte(s) or entheseophyte(s) formation on the tibia with bone remodeling or subchondral bone sclerosis or } \\
\text { femur condyle with new bone formation }\end{array}$ & 4 \\
\hline
\end{tabular}

(Scores adapted from Grauw et al. 2006 [8], Verwilghen et al. 2009 [9]).

abnormal radiographic changes or minor radiographic changes (82.86\%, $n=1538 / 1855$; grade $\leq 1)$. Only $2.9 \%(n=43 / 1855)$ of the joints were classified as grade 3 or 4 , corresponding to the presence of more severe radiographic changes.

The most affected joint (considering grade $\geq 1$ ) was TMT, where $89.28 \%$ of the joints presented some radiographic changes (Table 2). On the other hand, FTP was the least affected joint, with $97.87 \%$ of the joints considered as normal ( $n=45 / 47$, grade 0 ). Severe lesions (grade 3 and 4) were more frequently seen in TMT and, to a lesser extent, in DIT joints (Table 2).

The distribution of grades for each joint evaluated in each limb and the total for the forelimbs and hindlimbs are presented in Table 3.

\subsubsection{Distal Interphalangeal (DIP)}

Of 98 horses with DIP radiographs, $63.06 \%$ of the joints ( $n=246$ ) 390 ) had a grade $\geq 1$. Specifically, $45.13 \%(n=176 / 390)$ of the joints had grade $1,17.67 \%(n=69 / 390)$ had grade $2,0.26 \%(n=1 / 390)$ had grade 3 , and none presented changes of grade 4 (Table 2 ).

\subsubsection{Proximal Interphalangeal (PIP)}

For PIP radiographs, $73.92 \%(n=289 / 391)$ had a grade $\geq 1$ (Table 2$)$. The majority of the affected joints were classified as grade $1(49.11 \%, n=192 / 391)$, whereas $22.7 \%(n=89 / 391)$ were classified as grade 2 and $2.05 \%(n=8 / 391)$ as grade $\geq 3$.

The PIP radiographic changes were the second most common finding recorded in the forelimbs (70.92\%, $\mathrm{n}=139 / 391)$ (Table 3$)$.

\subsubsection{Metacarpo or metatarsophalangeal (MCP or MTP)}

The prevalence of MCP and MTP changes in fetlock radiographs that met the criteria for inclusion was high, with $57.66 \%$ ( $n=226$ / 392 ) considered as grade $\geq 1$, with most of these changes classified as grade $1(43.37 \%)$.

The radiographic changes in the MCP were the most common radiographic finding recorded in the forelimbs, with $71.34 \%$ ( $\mathrm{n}=$ $140 / 196$ ) of the joints with a grade $\geq 1$ (Table 3 ). When comparing the prevalence of radiological changes between MCP/MTP, the prevalence was higher in the forelimbs $(71.34 \% \mathrm{n}=140 / 196$, grade $\geq 1$ ) than in the hindlimbs $(43.88 \% n=86 / 196$, grade $\geq 1$ - Table 3$)$.

\subsubsection{Tarsi}

Radiographic changes in TMT joint were the most common finding recorded, and they occurred in $89.28 \%(n=175 / 196)$ of the joints evaluated (Table 2). This joint presented the highest prevalence of severe radiographic changes, with $8.16 \%(n=16 / 196)$ of the joints presenting changes of grade 3 and 4 (Tables 2 and 3 ). Considering the DIT, 67.86\% ( $n=133 / 196)$ were classified as grade $\geq 1$, with $51.02 \%$ of the joints presenting changes of grade 1 (Table 2 ). Only $18.37 \%(n=36 / 196)$ of the PIT/TT joints were classified as grade $\geq 1$, and no joints were classified as grades 3 and 4 (Tables 2 and 3 ).

\subsubsection{Stifle}

From the 47 horses that presented radiographs of the FTP, the vast majority presented no signs of lesion $(97.87 \%, n=45)$ with only 2 horses $(2.13 \%)$ presenting a minor radiographic change on the lateral trochlear ridge. Overall, the FTP had a very low prevalence of radiographic abnormalities (Tables 2 and 3 ).

\subsection{Correlation Between Radiographic Changes in Different Joints}

\subsubsection{Correlations Between the Same Joints in the Contralateral Limbs}

When analyzing the same joint contralaterally, a strong correlation was systematically found for all joints, with a correlation coefficient in the range of $0.6-0.7(P<.0001)$ (Table 4), except for the fetlock of the hindlimbs where the correlation was somewhat lower $(r=0.41, P<.0001)$. In the forelimbs, the highest correlation was found for MCP joints $(r=0.66, P<.0001)$, whereas in the hindlimbs, the highest correlation was for DIT joints $(r=0.71, P<$ .0001).

\subsubsection{Correlations Between the Same Joints in Front and Hindlimbs}

When comparing the radiographic scores for the same distal joint in the forelimbs and hindlimbs (Table 4), a weak positive correlation was found between the grade of the radiographic findings for DIP $(r=0.33, P<.001)$ and a moderate positive correlation for MCP/MTP joints $(r=0.48 P<.0001)$ and PIP joints $(r=$ $0.40, P<.0001)$.

\subsubsection{Correlations Between Different Joints on the Same Limb}

In the forelimbs, the existence of radiographic findings in the PIP joints was weakly/moderately correlated with the existence of findings in the fetlock of the same $\operatorname{limb}(r=0.34$ to $0.40, P<.01)$ (Table 5), whereas in the hindlimbs, a weak correlation was found between DIP and PIP of the same limb $(r=0.26$ to $0.37, P<.01)$. In addition, weak correlations were found in the tarsi, namely DIT with TMT $(r=0.22$ to $0.28, P<.05)$ and PIT $(r=0.36$ to $0.32, P<.01)$.

\subsubsection{Correlations Between Ipsilateral and Diagonal Limbs}

Concerning the distal limb (DIP, PIP and MCP/MTP) and tarsi (PIT/TT, DIT, TMT) (Table 6), strong associations were found between the contralateral limbs, with strong correlations between the contralateral distal forelimbs $(r=0.67, P<.0001)$, contralateral distal hindlimbs $(r=0.61, P<.0001)$, and tarsi $(r=0.72, P<.001)$.

For the diagonal distal limbs (Table 6 ), a moderate correlation $(r=0.57, P<.0001)$ was found between the right forelimb $(\mathrm{RF})$ and left hindlimb (LH), whereas for the other diagonal (LF-RH), the correlation was weak but positive $(r=0.29, P<.001)$. On the other hand, ipsilateral distal limbs' scores were also moderately correlated (distal RF and distal RH with $r=0.48, P<.0001$ and distal $\mathrm{LF}$ with distal LH with $r=0.30, P<.01$ ). 

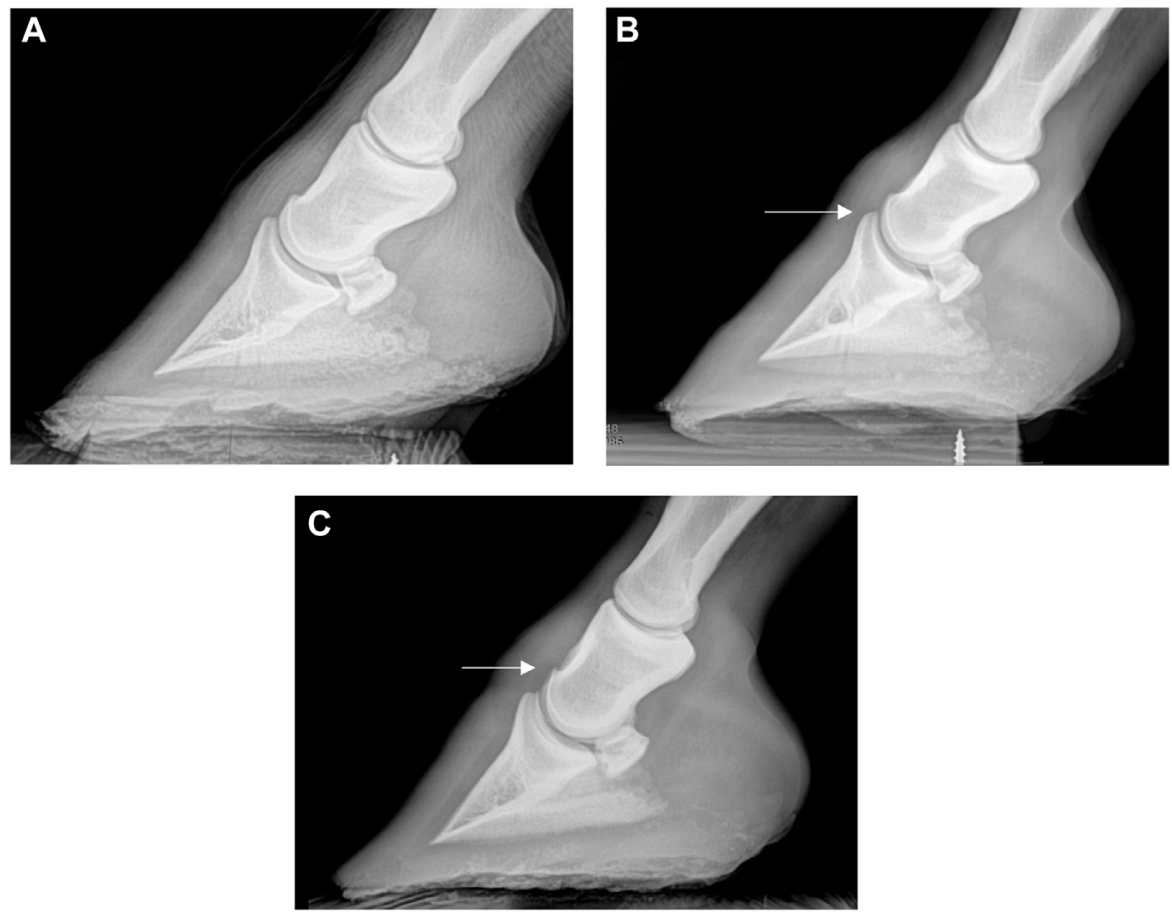

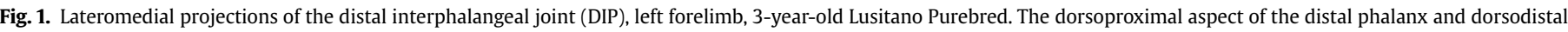

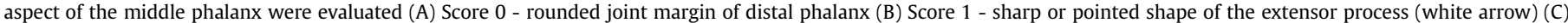

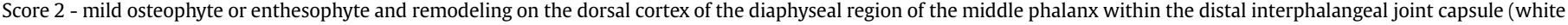
arrow).

The other correlations were less consistent between the limbs, but generally indicated a positive association between scores for the various joints.

\subsection{Effect of Age on $O A$}

The effect of age on the total score and the score attributed to the different joints was evaluated, and the significant regression equations $(P<.05)$ expressing the relationship of age with the severity of radiographic changes in various joints are shown in Fig. 5.

The total score increased, on average, by $0.213 \pm 0.092$ per year of age (Supplementary Table 1 ), revealing that the age can be a statistically significant predictor of radiographic changes. The individual joints where age had a significant effect were PIP $(P<$ $.0001)$ in the forelimbs and fetlock in the fore and hindlimbs $(P<$ .01 ), where the increase with age ranged between nearly 0.03 and 0.05 score points per year of age. The exception to this general pattern was detected in TMT of the $\mathrm{LH}$, where a decline with the age was observed.

\section{Discussion}

This study is the first comprehensive report describing the radiographic changes observed in the distal limb, tarsi, and stifle in the Lusitano Purebred. Studies with other breeds successfully used a radiographic scoring system that could reflect the severity of lesions and correlate them with soundness and future performance of the horse $[3,5,13-15]$. Our study, however, aimed to verify the radiographic prevalence and analyze the correlation between radiographic findings in the different limbs and joints and not the clinical prevalence itself. The use of a standardized objective scale in the evaluation of the radiographs allows the comparison of our results with those from other studies and can be used as a baseline indicator for further studies in the Lusitano Purebred horse.

Despite most of the joints presented some radiographic findings (59.63\% of the joints were classified as having some degree of change, $\geq 1$ ), the most frequent radiographic changes were in grade 1 for all joints (42.56\%, $\mathrm{n}=790 / 1855$ Table 2). If a score/grade 1 (Figs. 1-3) was not deemed a radiographic lesion with clinical significance, as many authors consider some of these changes to be minor, then the prevalence of clinically significant lesions (i.e., joints with grade $>1$ ) would be reduced to only $17.07 \%$ (Table 2 ). The fact that the majority of the population presented for radiographic examination in our study was represented by young sport horses may contribute to the low prevalence observed when compared with other studies where around $30 \%$ of all equine patients had intra-articular lesions related to OA [2] or nearly $88.9 \%$ of the horses presented some type of radiographic changes (excluding the normal variations of anatomy) [3]. Nevertheless, some of the inclusion criteria differed between studies, which make these comparisons difficult to carry out.

In our study, the TMT joint presented the highest prevalence of radiographic changes $(89.28 \%, n=175 / 196)$, but for the vast majority of the cases, these were classified as minor radiographic changes. The differentiation between osteophytes and entheseophytes at the dorsoproximal aspect of the third metatarsal bone (MtIII) in the TMT joint represented a challenge, as indicated by previous authors $[7,16]$. The osseous spurs on the proximal dorsal and dorsolateral aspects of the MtIII are mentioned in a previous study [7], whereas other studies mention the dorsomedial aspects of the DIT and TMT as a predominant location in horses with predisposition for OA [1]. It was interesting to verify that still regarding the tarsi, the PIT/TT joints were affected the least and the TMT (Fig. 4) and DIT (Fig. 4) were affected with the highest grades, indicating severe lesions, as it has been shown in previous studies $[6,17]$, where it is common to report that DIT and TMT are affected 

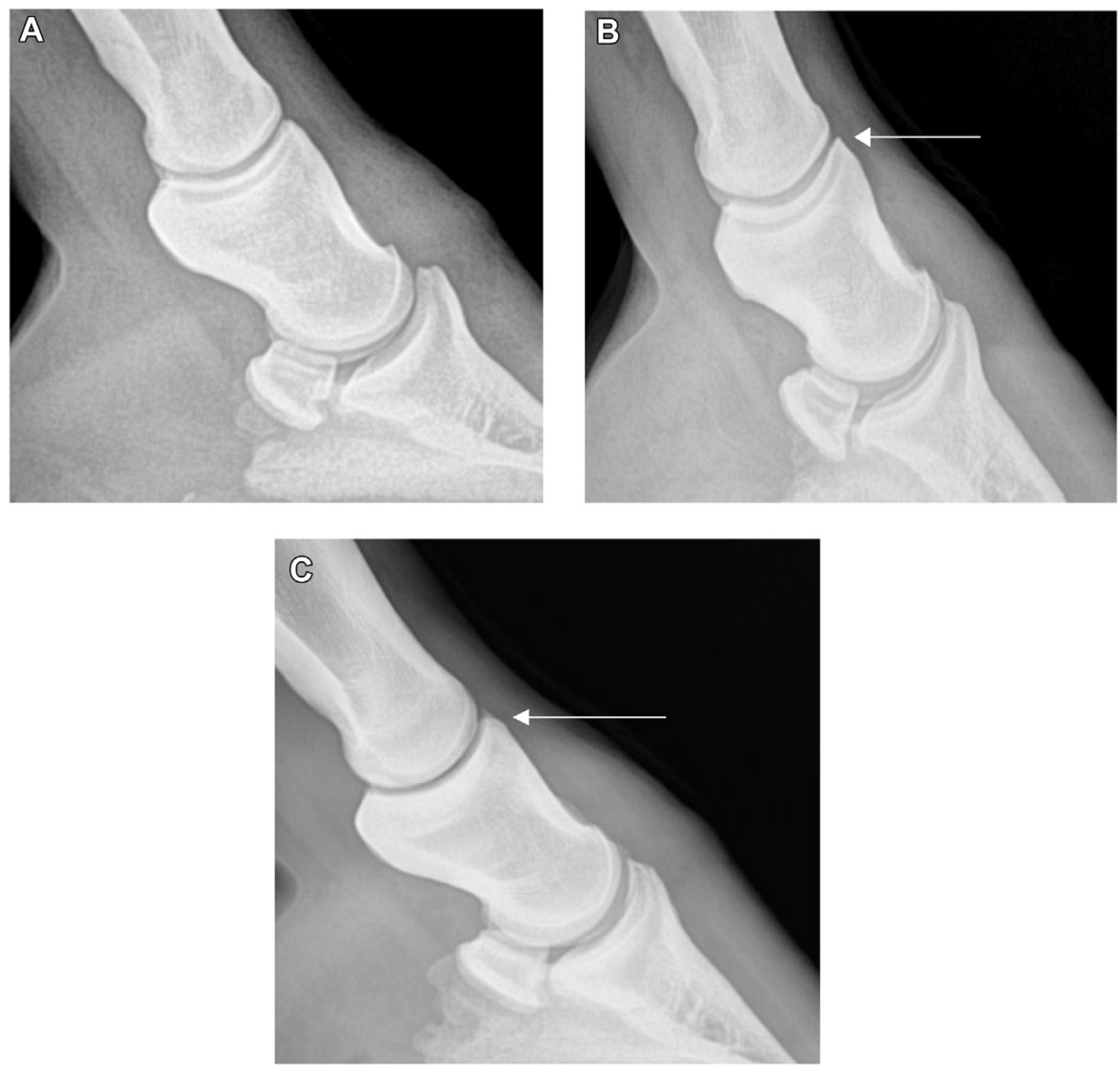

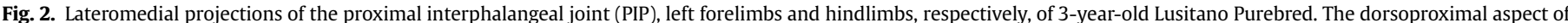

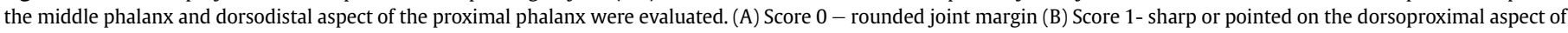
the middle phalanx (white arrow) (C) Score 2 - mild periarticular osteophyte on the dorsoproximal aspect of the middle phalanx (white arrow).

most frequently either alone or in combination [18]. Our results indicate a weak positive and significant correlation between the occurrence of OA in the contralateral joints of TMT and DIT, and this interesting observation is also supported by the literature that mentions that these two joints often present OA bilaterally [16,17]. It has been suggested that small articular areas, such as DIT/TMT, which are described as high load-low motion joints, are subjected to compressive forces and must sustain the same weight-bearing load for a relatively longer period of time during joint movement when compared with higher range motion joints, such as, for example, the TT $[1,6]$. Therefore, this may be the explanation for the low prevalence of abnormal radiographic findings in the TT joint. Although PIT is a low-motion joint, it communicates consistently with the TT [19], and for this reason, they were considered in the evaluation together. The OA in the PIT joint is reported as being rare and when present it seems to be accompanied with clinical signs, along with the TT joint [20].

The second most affected joint with OA was the PIP joint (Fig. 2). The predominant abnormal radiographic finding in the PIP joint was a periarticular osteophyte (Fig. 2) that, particularly in the hindlimb, can be an incidental or a relatively minor finding if there are no other OA signs associated. Nevertheless, similarly to the low motion joints of the tarsi, the PIP joint also presents a small crosssectional area that is responsible for supporting a large vertical load which predisposes more to injury [21] and probably for this reason, this joint appears in second place of the most affected.

The DIP joint was the fourth most affected joint with $63.06 \%$ ( $n=246 / 390$ ) when analyzing the prevalence of osteoarthritic radiographic findings. The most common finding is visible in the lateromedial view [22], and in our study, it corresponded to the modeling or presence of spurs in the extensor process of the distal phalanx, as well as at the dorsodistal aspect of the middle phalanx within the joint limits (Fig. 1). Another sign of articular cartilage damage is the narrowing of the joint space visible on the DP view that does not become radiographically evident until severe changes have occurred, turning this joint a challenge to evaluate and diagnose in the early stages of the disease [21]. The extensor process has to be carefully analyzed by the clinician in the lateromedial view as this region has normal anatomic variation of shape and size of the extensor process [18]. Comparison with the opposite limb can be beneficial to differentiate normal anatomic variation versus periarticular osteophyte formation [21]. The common digital extensor tendon inserts on the extensor process of the distal phalanx in the DIP joint and also entheseophytes need to be distinguished from osteophytes. Ultrasonographic examination was not contemplated in this study, and a differentiation between osteophyte and entheseophyte was not performed. However, the evaluation of this joint through the different imaging techniques has been previously studied, and according to Rovel et al. (2019) [23], higher grades are attributed with radiographic and ultrasonographic interpretation when comparing the findings present in the DIP with magnetic resonance imaging.

In the present study, the FTP joint showed a low prevalence of radiographic changes (97.9\% had grade 0 ) when it comes to the horses that compromised this joint in the radiographic protocol. Despite the lack of stifle radiographic projections in our study (such as caudocranial), it seems that the OA observations in Lusitano horses are similar to other breeds, such as the American Quarter 

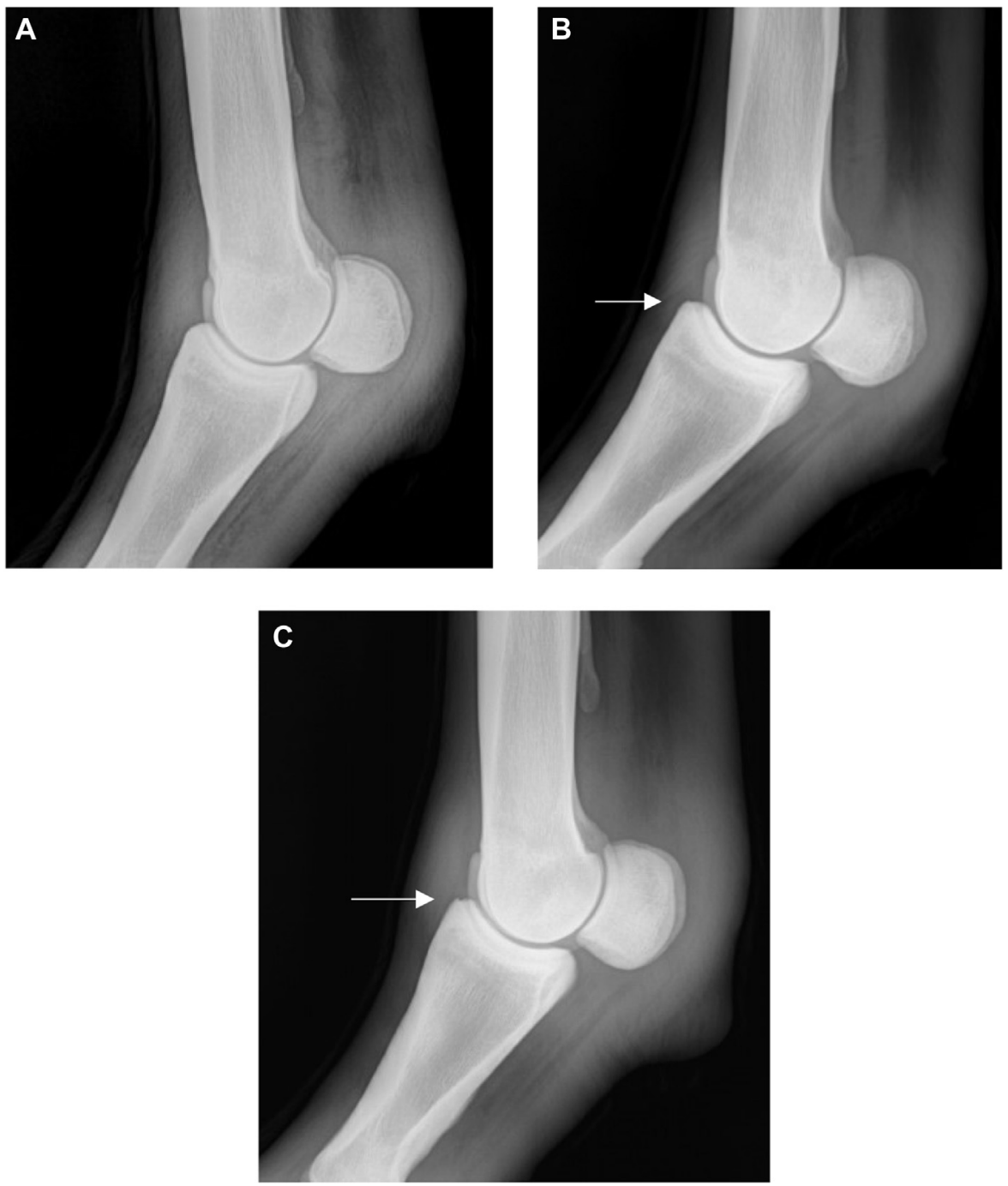

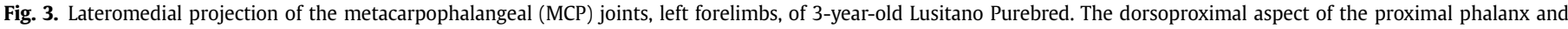

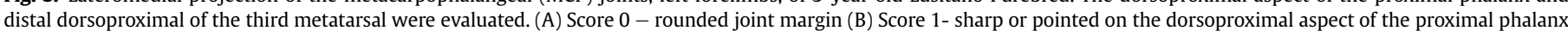
(white arrow) (C) Score 2 - mild periarticular osteophyte on the dorsoproximal aspect of the proximal phalanx (white arrow).

Horse, where even with more views the prevalence of OA was still low [3]. Although Contino el al. (2012) considered several other radiographic changes besides $\mathrm{OA}$, when radiographic changes compatible with $\mathrm{OA}$ in this study were considered (such as tibial osteophytes), only 6 of 454 (1.3\%) presented radiographic changes.

Although the relationship between radiographic findings and clinical evaluation was not assessed in the present study, it is important to mention that the correlation between the degree of radiological changes associated with $\mathrm{OA}$ and the degree of pain and lameness may not be very strong [24]. In addition, the mechanism and impact of OA is different in high and low motion joints [21], such that animals that have lameness associated with radiographic changes of OA in high motion joints (fetlocks and TT) often have subtle radiographic signs regarding osteophyte formation and subchondral bone lysis when compared with low motion joints such as the DIT, TMT, and PIP joints [25]. Signs of OA, including the size of osseous spurs at the dorsoproximal aspect of the MtIII, have been shown not to be of major clinical significance, with limited impact on the presence or severity of lameness [7]. Other mild to severe radiographic changes such as mild narrowing of the joint space and subchondral bone sclerosis in DIT and TMT usually lead to increased horse lameness and sometimes do not respond to intra-articular analgesia due to the subchondral pain [20].

When analyzing only the forelimbs, the joint with the highest prevalence of radiographic changes was the MCP joint, followed by the PIP joint. The common radiographic findings associated with $\mathrm{OA}$ in this joint are periarticular osteophytes and modeling of the proximodorsal aspect of the proximal phalanx (Fig. 3) that can be seen either in lateromedial and/or dorsopalmar and oblique views $[11,22]$, as it was observed in our sample of Lusitano horses. A study performed in race horses recognized the fetlock in the forelimbs as the major affected joint with the largest number of unique degenerative and traumatic lesions [12,26]. Another study performed with wild horses demonstrated that OA of the MCP joints occurs naturally, with lesions similar to the ones described in race horses [27]. Other studies mention that the extension angle of the fetlock during exercise (mainly at trot) can be correlated with injury, but currently, there is no evidence to specify the magnitude or frequency of hyperextension necessary to increase this risk [28]. However, caution is needed when comparisons are made between breeds, with some studies [29] reporting that different breed populations with different gait characteristics, such as the 

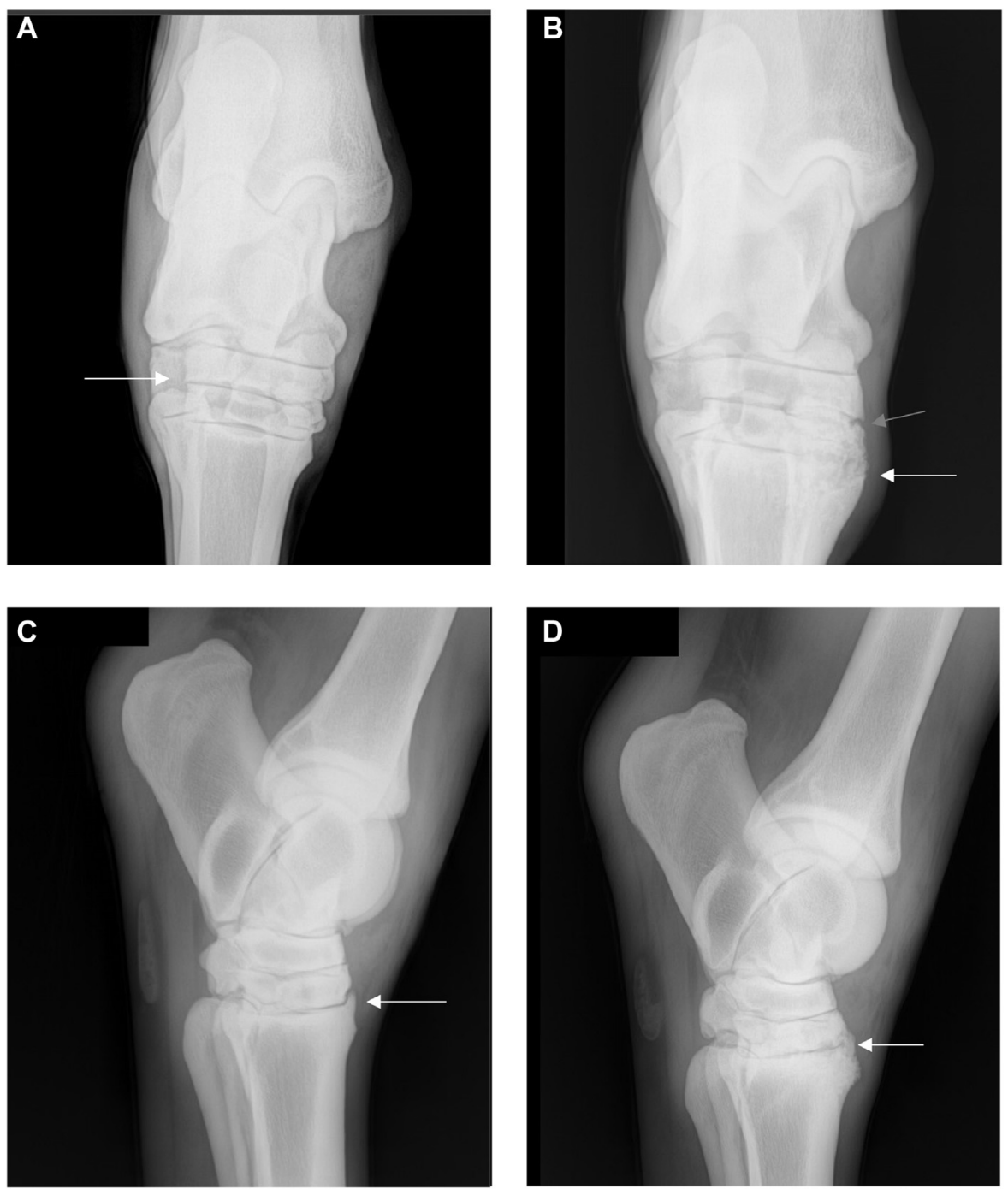

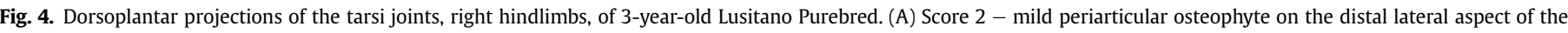

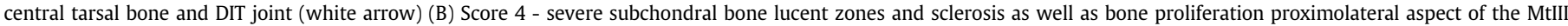

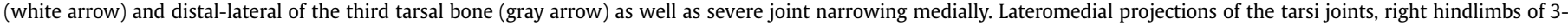

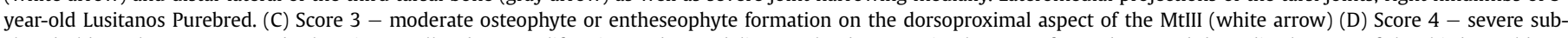

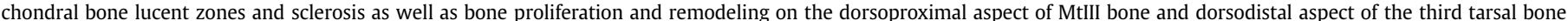
(white arrow).

Andalusian and Lusitano, should be compared with caution to other breeds.

The strong positive correlation between grades attributed to bilateral joints was validated in this study. These results agree with the current literature recommendations, which assume that it is not uncommon to have bilateral disease with either acute or chronic onset, and for that reason, it is recommended to perform radiographs of the contralateral joint for comparison $[1,18]$.

Table 2

Prevalence of radiographic changes by grade and joint $(\mathrm{n}=1855)$.

\begin{tabular}{|c|c|c|c|c|c|c|c|c|c|}
\hline \multirow[t]{2}{*}{ Grade $^{a}$} & \multicolumn{2}{|c|}{ Total prevalence } & \multicolumn{6}{|c|}{ Prevalence by joint (\%) } & \multirow[b]{2}{*}{ FTP } \\
\hline & $\mathrm{n}$ & $\%$ & DIP & PIP & MCP or MTP & TMT & DIT & PIT or TT & \\
\hline 0 & 748 & 40.30 & 36.95 & 26.08 & 42.35 & 10.72 & 32.14 & 81.63 & 97.87 \\
\hline 1 & 790 & 42.56 & 45.13 & 49.11 & 43.37 & 60.20 & 51.02 & 16.33 & 2.13 \\
\hline 2 & 274 & 14.76 & 17.67 & 22.77 & 11.48 & 20.92 & 13.27 & 2.04 & 0 \\
\hline 3 & 32 & 1.72 & 0.26 & 1.79 & 2.30 & 5.61 & 2.04 & 0 & 0 \\
\hline 4 & 11 & 0.59 & 0 & 0.26 & 0.51 & 2.55 & 1.53 & 0 & 0 \\
\hline$\geq 1$ & 1107 & 59.63 & 63.06 & 73.92 & 57.65 & 89.28 & 67.86 & 18.37 & 2.13 \\
\hline$\geq 2$ & 317 & 17.07 & 17.92 & 24.81 & 14.29 & 29.08 & 16.84 & 2.04 & 0 \\
\hline
\end{tabular}

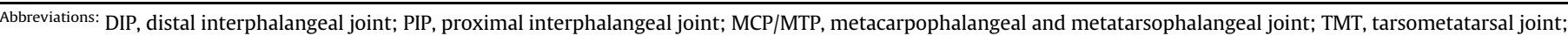
DIT, distal intertarsal joint; PIT/TT, proximal intertarsal and tibiotarsal joints; FTP, femorotibial-patellar joints.

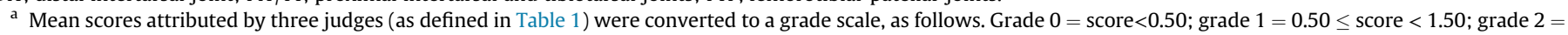
$1.50 \leq$ score $<2.50$; grade $3=2.50 \leq$ score $<3.50$; grade $4=3.50 \leq$ score . 
Table 3

Prevalence of radiographic changes of the distal limb joints, tarsi and stifle $(\mathrm{n}=1855)$.

\begin{tabular}{|c|c|c|c|c|c|c|c|}
\hline Joint & Grade $^{a}$ & LF \% (n) & $\mathrm{RF} \%(\mathrm{n})$ & LH \% (n) & $\mathrm{RH} \%(\mathrm{n})$ & Total forelimbs \% (n) & Total hindlimbs \% (n) \\
\hline \multirow[t]{5}{*}{ DIP } & 0 & $28.57(28)$ & $34.69(34)$ & $38.14(37)$ & $46.39(45)$ & $31.63(62)$ & $42.27(82)$ \\
\hline & 1 & $41.84(41)$ & $48.98(48)$ & $49.48(48)$ & $40.21(39)$ & $45.41(89)$ & $44.85(87)$ \\
\hline & 2 & $29.59(29)$ & $16.33(16)$ & $11.34(11)$ & $13.34(13)$ & $22.96(45)$ & $12.37(24)$ \\
\hline & 3 & $0(0)$ & $0(0)$ & $1.03(1)$ & $0(0)$ & $0(0)$ & $0.52(1)$ \\
\hline & 4 & $0(0)$ & $0(0)$ & $0(0)$ & $0(0)$ & $0(0)$ & $0(0)$ \\
\hline \multirow[t]{5}{*}{ PIP } & 0 & $29.59(29)$ & $28.57(28)$ & $21.43(21)$ & $24.74(24)$ & $29.08(57)$ & $23.09(45)$ \\
\hline & 1 & $51.02(50)$ & $50.51(49)$ & $44.90(44)$ & $50.52(49)$ & $50.51(99)$ & $47.71(93)$ \\
\hline & 2 & $17.35(17)$ & $18.37(18)$ & $31.63(31)$ & $23.71(23)$ & $17.86(35)$ & $27.67(54)$ \\
\hline & 3 & $2.04(2)$ & $3.06(3)$ & $1.02(1)$ & $1.03(1)$ & $2.55(5)$ & $1.03(2)$ \\
\hline & 4 & $0(0)$ & $0(0)$ & $1.02(1)$ & $0(0)$ & $0(0)$ & $0.51(1)$ \\
\hline \multirow[t]{5}{*}{ MCP or MTP } & 0 & $32.65(32)$ & $24.49(24)$ & $59.18(58)$ & $53.06(52)$ & $28.57(56)$ & $56.12(110)$ \\
\hline & 1 & $45.92(45)$ & $55.10(54)$ & $31.63(31)$ & $40.82(40)$ & 50.51 (99) & $36.23(71)$ \\
\hline & 2 & $18.37(18)$ & $14.29(14)$ & $8.16(8)$ & $5.10(5)$ & $16.33(32)$ & $6.63(13)$ \\
\hline & 3 & $3.06(3)$ & $6.12(6)$ & $0(0)$ & 0 & $4.59(9)$ & $0(0)$ \\
\hline & 4 & $0(0)$ & $0(0)$ & $1.02(1)$ & $1.02(1)$ & 0 & $1.02(2)$ \\
\hline \multirow[t]{5}{*}{ TMT } & 0 & & & $14.29(14)$ & $7.14(7)$ & & $10.72(21)$ \\
\hline & 1 & & & $60.20(59)$ & $60.20(59)$ & & $60.20(118)$ \\
\hline & 2 & & & 17.35 (17) & $24.49(24)$ & & $20.92(41)$ \\
\hline & 3 & & & $5.10(5)$ & $6.12(6)$ & & $5.61(11)$ \\
\hline & 4 & & & $3.06(3)$ & $2.04(2)$ & & $2.55(5)$ \\
\hline \multirow[t]{5}{*}{ DIT } & 0 & & & $35.71(35)$ & $28.57(28)$ & & $32.14(63)$ \\
\hline & 1 & & & $53.06(52)$ & $48.98(48)$ & & $51.02(100)$ \\
\hline & 2 & & & $7.14(7)$ & 19.39 (19) & & $13.27(26)$ \\
\hline & 3 & & & $3.06(3)$ & $1.02(1)$ & & $2.04(4)$ \\
\hline & 4 & & & $1.02(1)$ & $2.04(2)$ & & $1.53(3)$ \\
\hline \multirow[t]{5}{*}{ PIT/TT } & 0 & & & $83.67(82)$ & $79.59(78)$ & & $81.63(160)$ \\
\hline & 1 & & & $15.31(15)$ & $17.35(17)$ & & $16.33(32)$ \\
\hline & 2 & & & $1.02(1)$ & $3.06(3)$ & & $2.04(4)$ \\
\hline & 3 & & & $0(0)$ & $0(0)$ & & $0(0)$ \\
\hline & 4 & & & $0(0)$ & $0(0)$ & & $0(0)$ \\
\hline \multirow[t]{5}{*}{ FPT } & 0 & & & $97.9(46)$ & $97.87(46)$ & & $97.87(92)$ \\
\hline & 1 & & & $2.13(1)$ & $2.13(1)$ & & $2.13(2)$ \\
\hline & 2 & & & $0(0)$ & $0(0)$ & & $0(0)$ \\
\hline & 3 & & & $0(0)$ & $0(0)$ & & $0(0)$ \\
\hline & 4 & & & $0(0)$ & $0(0)$ & & $0(0)$ \\
\hline
\end{tabular}

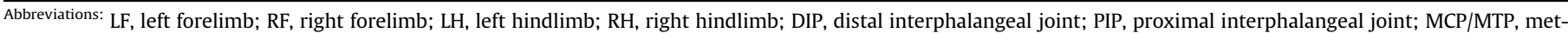

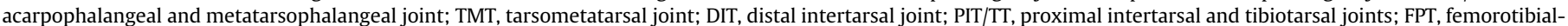
patellar joints.

a Grades as defined in Table 1.

In the forelimbs, moderate/weak correlations $(r=0.34-0.40)$ were observed between scores of the PIP and MCP joints, which may be related to the conformation of the distal limb $[21,30]$. Concerning the correlations between the scores of the tarsi joints, they were only significant between TMT and DIT and between DIT and PIT. Our study is in agreement with previous studies of tarsi that reported that these joints can be predominantly affected [6]

Table 4

Correlations between prevalence of radiographic changes in different joints.

\begin{tabular}{lll}
\hline Limbs & Joint & Correlation $^{\text {a }}$ \\
\hline Contralateral forelimbs & DIP & $0.63 * * *$ \\
& PIP & $0.65 * * *$ \\
Contralateral hindlimbs & MCP/MTP & $0.66 * * *$ \\
& DIP & $0.52^{* * *}$ \\
& PIP & $0.62^{* * *}$ \\
& MCP/MTP & $0.41^{* * *}$ \\
& TMT & $0.57 * * *$ \\
Forelimbs/hindlimbs & DIT & $0.71^{* * *}$ \\
& PIT/TT & $0.68 * * *$ \\
& DIPF/DIPH & $0.33^{* *}$ \\
& PIPF/PIPH & $0.40^{* * *}$ \\
& MCP/MTP & $0.48 * * *$ \\
\hline
\end{tabular}

Abbreviations: DIP, distal interphalangeal joint; PIP, proximal interphalangeal joint; MCP/MTP, metacarpophalangeal and metatarsophalangeal joint; TMT, tarsometatarsal joint; DIT, distal intertarsal joint; PIT/TT, proximal intertarsal and tibiotarsal joints; DIPF, distal interphalangeal joint forelimb; DIPH, distal interphalangeal joint hindlimb; PIPF, proximal interphalangeal joint forelimb; PIPH, proximal interphalangeal joint hindlimb.

a $* * * P<.001 ; * * P<.01 ; * P<.05$. and also with studies which found positive moderate correlations between the occurrence and the grade of OA for TMT and DIT and DIT and PIT [17].

Table 5

Correlations between prevalence of radiographic changes in different joints of the same limb. ${ }^{a}$.

\begin{tabular}{llcc}
\hline Limbs & Correlation & Left & Right \\
\hline Forelimb & DIP/PIP & 0.13 & $0.21^{*}$ \\
& DIP/FET & -0.03 & -0.19 \\
Hindlimb & PIP/FET & $0.40^{* * *}$ & $0.34^{* *}$ \\
& DIP/PIP & $0.26^{* *}$ & $0.37^{* * *}$ \\
& DIP/FET & -0.07 & 0.08 \\
& PIP/FET & 0.02 & 0.16 \\
DIP/TMT & 0.004 & 0.16 \\
DIP/DIT & -0.04 & 0.10 \\
& DIP/PIT & 0.08 & 0.14 \\
PIP/TMT & 0.04 & 0.09 \\
& PIP/DIT & -0.01 & 0.11 \\
& PIP/PIT & -0.01 & $0.2^{*}$ \\
FET/TMT & -0.13 & -0.06 \\
& FET/DIT & -0.11 & 0.05 \\
FET/PIT & -0.04 & 0.09 \\
TMT/DIT & $0.22^{*}$ & $0.28^{* *}$ \\
& TMT/PIT & 0.15 & 0.11 \\
DIT/PIT & $0.36^{* *}$ & $0.32^{* *}$ \\
\hline
\end{tabular}

Abbreviations: DIP, distal interphalangeal joint; PIP, proximal interphalangeal joint; FET, fetlock joint; TMT, tarsometatarsal joint; DIT, distal intertarsal joint; PIT/TT, proximal intertarsal and tibiotarsal joints.

a $* * * P<.0001 ; * * P<.01 ; * P<.05$. 
Table 6

Correlations between radiographic changes in distal extremity and tarsi ${ }^{\mathrm{a}}$.

\begin{tabular}{|c|c|c|c|c|c|c|}
\hline Joints & Distal limb LF & Distal limb RF & Distal limb LH & Distal limb RH & Tarsi LH & Tarsi RH \\
\hline Distal limb LF & - & $0.67 * * *$ & $0.30 * *$ & $0.29 * *$ & $0.23 * *$ & $0.30 * *$ \\
\hline Distal limb RF & & - & $0.57 * * *$ & $0.48 * * *$ & 0.09 & $0.20 *$ \\
\hline Distal limb LH & & & - & $0.61 * * *$ & -0.06 & 0.11 \\
\hline Distal limb RH & & & & - & 0.04 & $0.22 *$ \\
\hline Tarsi LH & & & & & - & $0.72 * * *$ \\
\hline Tarsi RH & & & & & & - \\
\hline
\end{tabular}

Abbreviations: $L F$, left forelimb; RF, right forelimb; $\mathrm{LH}$, left hindlimb; RH, right hindlimb.

a $* * * P<.0001 ; * * P<.01 ; * P<.05$.

Regarding the radiographic changes and the correlations of the distal limb with their ipsilateral and diagonal pairs, also to the best of our knowledge these have not been previously described, despite the common belief in clinical practice that compensatory lameness can be present both in the ipsilateral or contralateral limbs when a primary problem is present in the forelimb or hindlimb [31].

The age of horses included in our study ranged from 3 to 25 years, with a mean age of $7.45 \pm 5.64$ years. Despite the young age of the population, the mean radiological score increased with age in the PIP joint in the forelimbs (Fig. 5), revealing similarities with literature that describe older horses to be at a greater risk $[1,30]$, with the forelimbs being more frequently affected than the hindlimbs [30]. In humans and horses, a strong relationship is described between articular cartilage degeneration and increasing age [2], and it is generally accepted that the articular cartilage of the MCP joint of the horse is the most comparable with the human knee [32]. In this study, a significant increment of the radiological score was also registered in the forelimbs and hindlimbs of the fetlocks as age increased. In wild horses, cartilage degeneration is considered a naturally occurring process in older animals [27], and the age-related OA changes in the MCP joint have been associated with the increasing production of nonenzymatic glycation products, such as pentosidine cross-links, resulting in a stiffer collagen network of the extracellular cartilage matrix [33]. These nonenzymatic glycation have also been shown in humans to be related with this process and are associated with the cartilage degeneration observed as the age increases [34]. The degeneration of cartilage is variable and is not evenly distributed over the joint, and two factors have been proposed as triggers for the development of OA: biomechanical loading and age-related changes [35]. Surprisingly, the TMT joint of the LH did not correspond to the pattern found in other joints, as it is possible to observe in Fig. 5.

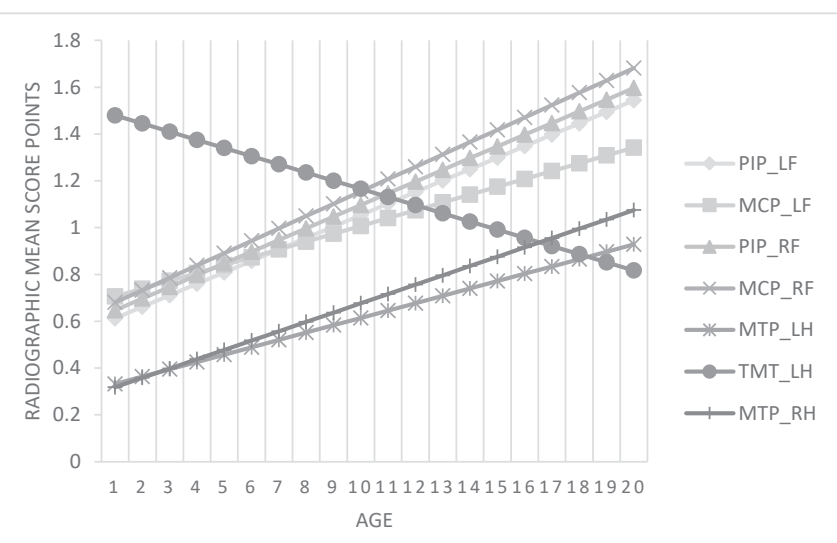

Fig. 5. Representation of regression equations for significant joint regression coefficients on age (years) in the analyses of prevalence of radiographic changes by joint.
The reason for this was that some horses revealed a high radiographic score (meaning severe lesions were observed in this joint) at a young age ( 3 year old), causing the unexpected relationship found between age and TMT lesions. The RH TMT joint also followed the same tendency as $\mathrm{LH}$, however, the values were not statistically significant and therefore were not reported in Fig. 5. In a study focused on the occurrence of osseous spurs on the articular margin of the dorsoproximal aspect of MtIII, Fairburn et al. [7] indicate that the severity of lesions is independent of age, which could justify a different relationship with age for this joint relative to others. Still, the high incidence of TMT lesions at a young age in our sample is of some concern and requires further investigation.

The present study had some limitations. The lack of flexed oblique, oblique (DIP and MCP/MTP), and caudocranial (FTP) radiographic views limited the viewing of periarticular osteophytes and joint space narrowing. In all stifles, the entire stifle was visible, and if needed, another projection would be taken as in some large horses the entire stifle cannot be included in a single image. The radiographs were repeated, to obtain a reasonably good quality image. The fact that an ultrasound evaluation was not performed is a limitation, as these could be important in detecting some periarticular spurs and differentiating osteophytes from enthesophytes, namely in the DIP, MCP/MTP, TMT, and FTP joints. The radiographic protocol was designed to obtain more information on a larger number of joints, taking into account the difficulties of examining young horses, while minimizing irradiation risks and limiting the costs by increasing the ability to detect radiographic changes on a given radiograph. The number of females in our study was low, but this reflects the fact that the group of animals analyzed was sampled among sport horses presented for radiographic exam, and these were mostly males. In fact, of the 324 Lusitano Purebred horses that participated in dressage competitions around Europe in 2016 and were included in the International Equestrian Federation ranks, only $22.6 \%$ were females [36].

In conclusion, this study demonstrates that there is a high prevalence of subtle radiographic signs of OA in Lusitano horses, but the clinical significance of these findings should be further investigated. Radiographic changes increased significantly with age in the PIP joints in the forelimbs and all fetlock joints. This study also confirms the clinical perception that OA findings are often present bilaterally in the distal limb and tarsi regions. Interestingly, positive correlations between OA signs in the distal high motion joints were found between forelimbs and hindlimbs and were also observed in the distal joints of the ipsilateral and the two diagonal limbs. To the authors' knowledge, these findings are reported for the first time in Lusitano horses and are potentially useful for veterinarians when assessing radiographic exams.

\section{Acknowlegment}

This work was partially supported by Project FEDER 2020 - 372 ALT20-03-0246-FEDER- 000019. 


\section{Supplementary data}

Supplementary data to this article can be found online at https://doi.org/10.1016/j.jevs.2020.103196.

\section{References}

[1] Kidd JA, Fuller C, Barr ARS. Tutorial article: osteoarthritis in the horse. Equine Vet Educ 2001;13:160-8.

[2] Orth MW, Schlueter AE, Orth MW. Equine osteoarthritis: a brief review of the disease and its causes. Equine Comp Exerc Physiol 2004;1:221-31.

[3] Contino EK, Park RD, Mc Ilwraith CW. Prevalence of radiographic changes in yearling and 2-year-old Quarter Horses intended for cutting. Equine Vet J 2012;44:185-95.

[4] Byam-Cook KL, Singer ER. Is there a relationship between clinical presentation, diagnostic and radiographic findings and outcome in horses with osteoarthritis of the small tarsal joints? Equine Vet J 2009;41:118-23.

[5] Couroucé-Malblanc A, Leleu C, Bouchilloux M, Geffroy O. Abnormal radiographic findings in 865 French Standardbred trotters and their relationship to racing performance. Equine Vet J 2006;38:417-22.

[6] Hoogmoed LM, Snyder JR, Thomas HL, Harmon FA. Retrospective evaluation of equine prepurchase examinations performed 1991-2000. Equine Vet J 2010;35:375-81.

[7] Fairburn A, Dyson S, Murray R. Clinical significance of osseous spurs on the dorsoproximal aspect of the third metatarsal bone. Equine Vet J 2010;42:591-9.

[8] Grauw J, Van de Lest C, Van Weeren R, Brommer H, Brama PAJ. Arthrogenic lameness of the fetlock : synovial fluid markers of inflammation and cartilage turnover in relation to clinical joint pain. Equine Vet J 2006;38:305-11.

[9] Verwilghen D, Busoni V, Gangl M, Franck T, Lejeune JP, Vanderheyden L, et al. Relationship between biochemical markers and radiographic scores in the evaluation of the osteoarticular status of Warmblood stallions. Res Vet Sci 2009;87:319-28.

[10] May S. Radiology refresher article. Equine Vet Educ 1996;8:114-20.

[11] Kawcak CE, Barrett MF. Fetlock. Joint Disease in the Horse. 2nd. Elsevier; 2016. p. $302-17$.

[12] Pool RR, Meagher DM. Pathologic findings and pathogenesis of racetrack injuries. Vet Clin North Am Equine Pract 1990;6:1-30.

[13] Robert C, Valette JP, Denoix JM. Correlation between routine radiographic findings and early racing career in French Trotters. Equine Vet J 2006;38:473-8.

[14] Cohen ND, Carter GK, Watkins JP, O'Conor MS. Association of racing performance with specific abnormal radiographic findings in Thoroughbred yearlings sold in Texas. J Equine Vet Sci 2006;26:462-74.

[15] Miyakoshi D, Senba H, Shikichi M, Maeda M, Shibata R, Misumi K. A retrospective study of radiographic abnormalities in the repositories for Thoroughbreds at yearling sales in Japan. J Vet Med Sci 2017;79:1807-14.

[16] Butler JA, Colles CM, Dysion SJ, Kold SE, Poulos P. The tarsus. Clin Radiol Horse 2017:329.

[17] Sprackman L, Dakin SG, May SA, Weller R. Relationship between the shape of the central and third tarsal bones and the presence of tarsal osteoarthritis. Vet J 2015;204:94-8.
[18] Butler JA, Colles CM, Dyson SJ, Kold SE, Poulos PW. General principles. Clinical Radiology of the Horse. 4th ed. Wiley-Blackwell; 2017. p. 15.

[19] Baxter G, Stashak T. Examination for lameness, Perineural and intrasynovial anesthesia. Lameness in Horses. Blackwell Publishing; 2011. p. $173-99$.

[20] Kawcak CE. Tarsus. Joint Disease in the Horse. 2nd ed. Elsevier; 2016. p. 347-53.

[21] Frisbie DD, Werpy NM, Kawcak CE, Barrett MF. Distal limb. Joint Disease in the Horse. 2nd ed. Elsevier; 2016. p. 281-301.

[22] Butler JA, Colles CM, Dysion SJ, Kold SE, Poulos P. Foot, pastern and fetlock. Clin Radiol Horse 2017:83-166.

[23] Rovel T, Coudry V, Jacquet-guibon S, Denoix J. Evaluation of standing low-field magnetic resonance imaging for diagnosis of advanced distal interphalangeal primary degenerative joint disease in horses: 12 cases (2010-2014). J Am Vet Med Assoc 2019;254:257-65.

[24] Dyson S, Ross M. Radiography and radiology. Diagnosis and Management of Lameness in the Horse. Lameness Horse; 2011. p. 204-5.

[25] May SA. Radiological aspects of degenerative joint disease. Equine Vet Educ $1996 ; 8: 114-20$

[26] Kane AJ, Park RD, McIlwraith CW, Rantanen NW, Morehead JP, Bramlage LR Radiographic changes in Thoroughbred yearlings. Part 1: prevalence at the time of the yearling sales. Equine Vet J 2003;35:354-65.

[27] Cantley CEL, Firth EC, Delahunt JW, Pfeiffer DU, Thompson KG. Naturally occurring osteoarthritis in the metacarpophalangeal joints of wild horses. Equine Vet J 1999;31:73-81.

[28] Walker VA, Tranquille CA, Newton JR, Dyson SJ, Brandham J, Northrop AJ, et al. Comparison of limb kinematics between collected and lengthened (medium/ extended) trot in two groups of dressage horses on two different surfaces. Equine Vet J 2017;49:673-80.

[29] Tranquille CA, Nankervis KJ, Walker VA, Tacey JB, Murray RC. Current knowledge of equine water treadmill exercise: what can we learn from human and canine studies? J Equine Vet Sci 2017;50:76-83.

[30] Baxter G. The pastern. In: Baxter G, editor. Adams and Stashak's Lamenesss in Horses. 6th ed. Wiley-Blackwell; 2011. p. 559.

[31] Ross M, Dyson S. Lameness in horses: basic facts before starting. Diagnosis and Management of Lameness in the Horse. 2nd ed. Elsevier Saunders; 2011. p. 3.

[32] Kuyinu EL, Narayanan G, Nair LS, Laurencin CT. Animal models of osteoarthritis: classification, update, and measurement of outcomes. J Orthop Surg Res 2016;11:1-27.

[33] Brama PAJ, Karssenberg D, Barneveld A, Weeren PR Van. Contact areas and pressure distribution on the proximal articular surface of the proximal phalanx under sagittal plane loading 2001;33:26-32.

[34] Verzijl N, Groot JDE, Oldehinkel E, Bank RA, Thorpe SR, Baynes JW, et al. Age related accumulation of Maillard reaction products in human articular cartilage collagen. Biochem J 2000;387:381-7.

[35] Brommer H, van Weeren PR, Brama PAJ, Barneveld A. Quantification and agerelated distribution of articular cartilage degeneration in the equine fetlock joint. Equine Vet J 2003;35:697-701.

[36] Bartolomé E, Milho S, Prazeres J. Genealogical and morphological analysis of Lusitano Purebred horses participating at international Dressage competitions. Res Vet Sci 2019;122:124-31. 